\title{
Regards croisés sur l'action des ONG en éducation au Sud
}

Table ronde

Differing viewpoints on NGOs' educational activities in the South. Round table

Miradas cruzadas sobre la acción de las ONG en educación en el Sur. Mesa

redonda

Sandra Barlet, Roland Biache, Mahfou Diouf, Bernard Dumont, André Gauron, Valérie Huguenin, Jacques Marchand et Gilbert Migan

\section{CpenEdition}

\section{Journals}

Édition électronique

URL : http://journals.openedition.org/ries/2180

DOI : $10.4000 /$ ries. 2180

ISSN : 2261-4265

Éditeur

Centre international d'études pédagogiques

Édition imprimée

Date de publication : 1 décembre 2011

Pagination : 57-66

ISBN : 978-2-85420-593-0

ISSN : 1254-4590

Référence électronique

Sandra Barlet, Roland Biache, Mahfou Diouf, Bernard Dumont, André Gauron, Valérie Huguenin, Jacques Marchand et Gilbert Migan, "Regards croisés sur l'action des ONG en éducation au Sud », Revue internationale d'éducation de Sèvres [En ligne], 58 | décembre 2011, mis en ligne le 01 décembre 2014, consulté le 02 mai 2019. URL : http://journals.openedition.org/ries/2180 ; DOI : 10.4000/ ries. 2180 


\title{
Regards croisés sur l'action des ONG en éducation au Sud
}

\section{Table ronde ${ }^{1}$}

\author{
Sandra Barlet \\ Roland Biache \\ Mahfou Diouf \\ Bernard Dumont \\ André Gauron \\ Valérie Huguenin \\ Jacques Marchand \\ Gilbert Migan
}

Cette table ronde porte sur les liens entre l'action des ONG en éducation et celle des pouvoirs publics. Elle réunit les regards différents et complémentaires de représentants d'ONG, de bailleurs, d'institutionnels au Sud, d'experts, et de croiser leurs analyses.

Gilbert Migan est chargé de la formation et de l'enseignement des jeunes au ministère de l'Enseignement secondaire, de la Formation technique et professionnelle, de la Reconversion et de l'insertion des jeunes du Bénin, où il coordonne un projet de promotion de l'enseignement technique et de la formation professionnelle (EFTP); Roland Biache, délégué général de Solidarité lä̈que, une ONG active notamment au Bénin, anime la commission Éducation et formation de Coordination Sud ${ }^{2}$; Mahfou Diouf, originaire du Sénégal, où il a travaillé pendant plusieurs années, ainsi que dans la sous-région ouestafricaine, travaille à Paris à la direction France-Europe d'Aide et Action, une ONG de solidarité internationale qui intervient dans vingt-neuf pays dans l'éducation de base et la formation, d'une manière générale; Bernard Dumont s'est toujours occupé d'éducation, à titre professionnel avec la coopération française à ses débuts, avec l'Unesco au Mali, au ministère français de l'Éducation où il a participé à la création de la formation professionnelle continue pour les adultes, pour l'Union européenne et pour la Banque mondiale, puis dans diverses associations; André Gauron, conseiller maître honoraire à la Cour des comptes et économiste de formation, intervient comme consultant pour le ministère des Affaires étrangères et européennes (MAEE), l'Agence française de développement (AFD) et le Centre international d'études pédagogiques (CIEP) en matière d'évaluation et de définition de

1. Cette table ronde s'est tenue le 30 septembre 2011 à l'Agence française de développement (AFD) à Paris. Elle a pu être organisée en visioconférence avec le Bénin grâce au concours de l'AFD-Paris et de l'AFD-Cotonou. La transcription a été assurée par Françoise Delinot et révisée par Marie-José Sanselme, avec Sandra Barlet.

2. Coordination Sud (Solidarité, Urgence, Développement) est la coordination nationale des ONG françaises de solidarité internationale. Rassemblant plus de centre trente ONG, elle assure une mission d'appui à leur professionnalisation et représente leurs positions auprès des institutions publiques et privées, en France et dans le monde. La commission Éducation et formation veille notamment à la bonne harmonisation des différentes initiatives prises en France dans le cadre de la campagne mondiale sur l'éducation. 
projet de formation professionnelle et il a également présidé, à la demande du MAEE, plusieurs groupes de travail sur la stratégie de la coopération française dans le domaine de l'éducation et de la formation professionnelle; l'AFD est représentée par Jacques Marchand, qui travaille à la Division éducation et formation professionnelle de l'AFD sur plusieurs pays, parmi lesquels Madagascar, le Togo, prochainement la Côte d'Ivoire, Haïti, Djibouti, dans le domaine de l'éducation principalement, ainsi que, dans une moindre mesure, dans celui de la formation professionnelle, et par Valérie Huguenin, de la Division des partenariats avec les ONG, où elle s'occupe du secteur, entre autres, de l'éducation. Sandra Barlet, enfin, travaille au Gret, une association française de professionnels de la solidarité internationale, active dans le domaine du développement et notamment de la formation et de l'insertion professionnelles des jeunes.

SANDRA BARLET : Je vous propose de répondre d'abord à un premier questionnement : «Quelle est, selon vous, l'évolution la plus marquante qui a caractérisé le monde des ONG ces dernières années dans le champ de l'éducation?». Vous pourrez illustrer vos réponses par des exemples représentatifs de leurs activités thématiques et de leurs positionnements. Puis nous aborderons la question de l'avenir : " quels nouveaux rôles se dessinent pour les ONG et à quels enjeux de développement éducatif devront-elles répondre à l'horizon 2020 ?»

Bernard Dumont : Avant d'aborder cette première question, je dirai en préalable que je préfère utiliser l'appellation «ASI ", association de solidarité internationale, car « ONG » a une connotation négative et vient d'ailleurs. Ensuite, on ne peut pas aborder un débat comme celui-là sans insister sur l'extrême diversité de ces ASI. Elles sont diverses par toutes sortes de critères - dimension, origine, financement, statut de leurs personnels - et il est impossible d'apporter une réponse globale aux questions posées, avec une telle diversité de situation.

SANDRa Barlet : C'est pourquoi notre analyse s'appuiera sur des exemples, car il est effectivement complexe de chercher à caractériser une réalité plurielle. Nous parlons de toutes les structures non gouvernementales, qu'elles soient du pays ou étrangères, locales ou internationales. C'est pour cela que nous avons conservé cette appellation "négative».

\section{D'UNE INTERVENTION VOLONTARISTE À LA MOBILISATION DE LA SOCIÉTÉ CIVILE}

Roland Biache: Les organisations actives dans le domaine de l'éducation interviennent dans l'espace que les organisations de la société civile (OSC) ont occupé peu à peu depuis vingt-cinq à trente ans, "à côté » des 
pouvoirs publics - dans certains cas en connivence forte, dans le meilleur sens du terme, et dans d'autres en opposition ou en alternative avec eux, dans un souci, malgré tout, de complémentarité très forte puisqu'elle est normée. En France, il existe un statut des associations complémentaires de l'École qui accompagnent le service public d'éducation et qui ont bien une démarche d'intérêt général, ce que l'on retrouve parfois au Sud, sans que ce soit tout à fait comparable. Un certain nombre d'organisations complémentaires de l'École ont été "nationalisées » aux indépendances, puisqu'elles étaient des sections nationales des associations de l'ex-puissance coloniale, et se sont transformées en ONG «locales ", notamment au Bénin, où elles sont bien connues, comme par exemple le Conseil des activités éducatives du Bénin (CAEB). Depuis, d'autres structures se sont mises en place dans la famille des organisations de la société civile au-delà des associations : il s'agit des coopératives, des mutuelles, des syndicats, etc., en résumé toutes celles qui participent du dialogue social.

Mahfou Diouf : On relève plusieurs évolutions. La plus marquante est un changement de démarche. Au départ, l'intervention des ONG était plutôt volontariste : il s'agissait de régler les problèmes qui se posaient notamment dans plusieurs pays du Sud, où les défis à relever étaient considérables. Cette approche a porté ses fruits mais les difficultés d'appropriation, par les populations, des projets et des politiques éducatives mis en place ont été très importantes. Cela a permis de faire évoluer la vision du développement de l'éducation à partir d'un nouveau postulat: pour que l'éducation soit l'affaire de tous, il faut travailler au renforcement de la capacité des acteurs. On est passé ainsi d'une approche volontariste à une approche d'accompagnement des populations. L'intervention actuelle des ONG vise plutôt à faire évoluer non seulement les systèmes mais aussi les mentalités, afin de faire prendre conscience des enjeux que recouvre l'éducation. C'est une intervention très complexe, car il est plus simple d'avoir des équipes sur le terrain mettant en œuvre des activités que d'adopter une posture où l'on ne se substitue pas à l'acteur mais où, au contraire, on essaie de faire en sorte que l'acteur puisse être maître de son processus. Les acteurs renforcés sont des associations de parents d'élèves, des associations locales, et ces organisations de la société civile dans les pays en voie de développement sont accompagnées, appuyées par des organisations de plus grande envergure, notamment du Nord, qui travaillent dans ces pays.

SANDra BARLET : Justement, quel est leur rôle par rapport aux États, dans ce travail de renforcement de la société civile?

Mahfou Diouf : On constate de nombreuses failles dans la gestion du système. La volonté de responsabiliser par exemple les associations de parents d'élèves existe, mais aucun travail n'est fait pour donner à ces populations les 
moyens d'agir. C'est là une niche d'intervention pour les ONG, qui agissent pour que les communautés, les collectivités locales puissent réellement maîtriser les enjeux liés à l'éducation. C'est aussi valable pour les corps d'inspection de l'éducation, qui ont la prérogative d'animer le dispositif au niveau local. Si ces structures ne sont pas accompagnées, la gestion du système en souffre. C'est vraiment l'évolution majeure aujourd'hui.

Bernard Dumont : J'approuve tout à fait ce qui vient d'être dit. Ce sont justement les associations de solidarité internationale qui ont, les premières, mis l'accent sur la mobilisation des acteurs autour de l'école. Aujourd'hui, les législations d'un certain nombre de pays prévoient des comités de gestion scolaire. Ces derniers n'ont pu apparaître qu'à partir des expériences menées par les associations pour former des associations de parents d'élèves et pour mobiliser la société civile, par exemple les artisans, et des personnalités autour de l'école. Ce que dit Mahfou Diouf est en voie de généralisation institutionnelle, mais il ne faut pas oublier le rôle qu'ont joué les ONG pour cela.

\section{D'UNE APPROCHE SECTORIELLE À LA GESTION LOCALE DE L'ÉdUCATION}

SANDra Barlet : Outre les évolutions dans les modes d'action, observet-on aussi de nouveaux champs d'intervention, de nouvelles thématiques?

Mahfou Diouf : Aujourd'hui, les thématiques sont plus larges car les ONG tiennent compte désormais de l'environnement de l'école. Après avoir beaucoup travaillé dans l'école, elles se sont aperçues que les changements n'étaient pas vraiment perceptibles et que l'environnement de l'école devait être pris en charge, c'est-à-dire les comités de gestion, comme l'a dit Bernard Dumont, ainsi que d'autres acteurs, et notamment les femmes, dont le rôle est essentiel pour la scolarisation des filles. Par exemple en Afrique de l'Ouest, elles se sont organisées en groupements et mènent des projets qui génèrent des revenus, réinvestis dans l'appui aux fournitures, au transport scolaire, etc.

Roland Biache: On est passé d'une intervention strictement sectorielle à une approche de type "développement local», y compris dans le processus d'accompagnement de la décentralisation, qui, dans de nombreux pays, est calquée sur la nôtre, avec une partie des mêmes prérogatives, voire la totalité, mais beaucoup moins de moyens. Autrement dit, on a déconcentré les responsabilités mais pas forcément les ressources humaines et les moyens financiers qui accompagnent cette décentralisation. Dans les pays d'Afrique de l'Ouest francophone, c'est caractéristique. Sur la question des publics, si l'on prend un 
sujet qui nous tient à cœur, à Solidarité laïque, comme à beaucoup d'autres, la petite enfance est totalement négligée ou privatisée et on sait qui cela touche - les classes aisées - ou ne touche pas - une partie des classes moyenne et les pauvres. Dans d'autres cas, il y a des projets d'OSC, que l'on peut classer dans le formel ou le non formel, et qui croisent ce que l'on appelle l'éducation tout au long de la vie, formant ainsi une sorte de continuum. Cette envie de continuum, qui est très intéressante, télescope par moments la formation professionnelle. Un enjeu majeur, dans les pays concernés par nos actions, autant du côté de l'AFD d'ailleurs que du côté des ONG, c'est cette espèce de "vide » qu'il y a dans le post-primaire : beaucoup de jeunes sortent du primaire avec un très faible bagage éducatif et se retrouvent sur le marché du travail sans qualification.

SANDra BARLET : Les ONG interviennent donc aux niveaux opérationnel et financier lorsqu'elles agissent là où les gouvernements ne peuvent aller, pour pallier les déficiences liées à la mise en place de la décentralisation, et au niveau thématique, lorsque certains champs, comme le post-primaire, ne sont pas suffisamment pris en charge et qu'elles jouent un rôle précurseur.

Bernard Dumont : Il ne s'agit pas seulement du post-primaire. Ce sont tous les besoins d'éducation tout au long de la vie qui ne sont pas satisfaits par l'école proprement dite et, notamment l'école primaire. Mais il faut penser à tous les adultes qui, encore aujourd'hui, ne sont pas allés à l'école, et à tous les domaines de l'alphabétisation fonctionnelle, soit générale, soit rapportée à une activité dans un projet. C'est un champ immense et qui est, à peu près partout, assuré par des associations. Certains États ont même prévu spécifiquement le rôle et la place des associations pour réaliser cet aspect-là de l'éducation, qu'on appelle non formelle et qui correspond à des besoins peut-être plus vastes que ceux de l'École proprement dite.

\section{QUELLE CONCEPTION DU Rôle DE L'ÉTAT ?}

André Gauron : Du point de vue de la coopération française, à partir de l'expérience que j'ai de l'évaluation ou de la définition de projets, on peut dire que les ONG sont plutôt présentes là où l'institutionnel ne l'est pas. La coopération française, par les relations qu'elle a avec les autorités locales, se situe plutôt dans une approche institutionnelle. Il s'agit d'appuyer des administrations, des dispositifs d'éducation et ce n'est qu'à la marge, plutôt récemment d'ailleurs, qu'on a vu apparaître dans certains projets une dimension de mobilisation des associations de parents d'élèves, qui n'existait pas dans des projets plus anciens. On observe une évolution vers une mobilisation des acteurs. Mais ce n'est pas la seule conception possible de l'appui que la coopération institutionnelle française pourrait fournir. Elle appuie des dispositifs mais d'autres bailleurs, notamment 
dans le post-primaire, l'apprentissage, la formation professionnelle, centrent davantage leur action sur les publics. Ainsi, il y a quelques années au Niger, pour bénéficier de financements pour des formations en apprentissage, l'Union européenne et la Banque mondiale avaient imposé de cibler des publics déscolarisés et non des élèves sortant du primaire et entrant dans le système de la formation professionnelle. Ce sont deux approches différentes. Ce qui est en jeu, c'est en fait la conception du rôle de l'État dans le domaine de l'éducation et de la formation professionnelle. Dans la vision très classique de la francophonie, très franco-française, l'État est à la fois celui qui conçoit la formation et celui qui gère les dispositifs de formation. Les rencontres récentes avec des pays anglo-saxons autour de la question des cadres nationaux de certification, notamment à Kampala et à l'Île de la Réunion, ont montré que si l'on se donne comme objectif les résultats qu'on doit atteindre en matière de scolarisation et de formation en centrant le rôle de l'État sur les contenus et sur les certifications, sans pour autant prendre en charge la gestion, on ouvre un espace assez grand. Cet espace concerne non seulement ceux qui sont déjà présents mais aussi les congrégations, qui sont extrêmement présentes dans un certain nombre de pays d'Afrique dans le domaine éducatif, ainsi que les ONG qui pourraient intervenir en tant que prestataires de formation, dès lors qu'elles acceptent les cadres définis par le pouvoir politique en termes de contenus, de contrôle, de résultats à atteindre.

\section{QUELS BESOINS EN INTERVENTION NON GOUVERNEMENTALE?}

Valérie Huguenin : En dix ans, l'évolution est visible. Elle concerne le renforcement des capacités, l'appui à la gestion locale. Certaines ONG restent très caritatives et cloisonnées mais cela devient plus rare. Beaucoup d'ONG, notamment celles du Nord, ont compris le rôle qu'elles pouvaient jouer auprès des partenaires du Sud en termes de structuration des associations locales, des communautés. Il s'agit de les aider à contribuer à la réflexion, à l'élaboration, au suivi des politiques publiques d'éducation. Même si les résultats sont en deçà des attentes des uns et des autres, les ONG du Nord jouent un rôle intéressant pour accompagner leurs partenaires dans ces nouveaux processus de dialogue avec l'État. Un autre apport très intéressant des ONG concerne les innovations, notamment pédagogiques. Les ONG portent parfois des actions pédagogiques très intéressantes, qu'elles promeuvent auprès des pouvoirs publics.

Bernard Dumont : Sur ce point, je pense que c'est le domaine où les associations jouent le rôle le plus important. Je prends un exemple : tous les gouvernements, toutes les lois d'orientation d'éducation, dans tous les pays, disent qu'il faut promouvoir les méthodes d'éducation active mais il y a fort peu 
de capacités nationales pour réaliser cette promotion. L'un des rôles les plus efficaces des associations de solidarité internationale du Nord est de contribuer à la mise en application de méthodes pédagogiques nouvelles et à la formation des acteurs en place, y compris par des formes assez originales comme l'appui à la création de regroupements d'enseignants, d'équipes pédagogiques qui s'organisent pour assurer elles-mêmes leur propre perfectionnement et qu'il faut aider à leurs débuts. Sans l'intervention des ONG, cela ne se ferait pas et c'est un champ d'intervention énorme qui s'ouvre aux activités de solidarité internationale.

André Gauron : Ce n'est pas aussi simple que cela, si je pense à ce qui s'est fait dans le domaine de la pédagogie de la rénovation des cursus. Les ONG interviennent, relativement ponctuellement, en expérimentation sur un champ géographique limité. Le problème de l'État pour réaliser les objectifs mondiaux de scolarisation, c'est celui de l'universalité et de la massification du dispositif. En règle générale, les États et les corps d'inspection des ministères souhaitent que les curricula et les méthodes pédagogiques soient les mêmes partout. Dans les pays de la zone francophone, on n'est pas dans une logique où, finalement, chacun peut, dès lors que la scolarisation est effectuée, développer les méthodes pédagogiques qu'il souhaite. On reste dans des cadres extrêmement normés avec des États qui veillent à une certaine unité pédagogique. Le secteur privé, qui ne relève pas des ONG, mais est soit de type confessionnel, soit de type commercial, joue un rôle relativement important. Il se coule cependant dans le moule étatique en matière de curricula, même si les méthodes peuvent différer et sont parfois plus intéressantes que celles du secteur public. Si l'on veut que les associations de solidarité locales nationales ou internationales jouent un rôle plus important, il faut arriver à articuler leur intervention dans le domaine pédagogique avec les objectifs que fixe l'État. Je crois qu'on restera encore longtemps dans un système où les États veulent un certain contrôle, voire une certaine uniformité des méthodes pédagogiques. Au Niger, qui a une frontière commune notamment avec la Libye, les autorités ont pris conscience qu'à travers des ONG d'origine libyenne ou saoudienne, un enseignement orienté d'un point de vue salafiste pouvait être introduit. Elles ont, en réaction, exprimé une volonté très claire de maîtriser les curricula, de façon à éviter ce type de dérive idéologique. L'influence d'un certain nombre de congrégations évangélistes d'origine américaine dans ces pays pose le même genre de problèmes. Il faut être attentif au fait ce que ce n'est pas seulement un problème éducatif. Il existe des enjeux idéologiques et politiques beaucoup plus importants qui justifient parfois que l'État reste très vigilant dans le domaine de la pédagogie.

Bernard Dumont : Je voudrais nuancer ce qui a été dit. Ce sont les gouvernements eux-mêmes qui souhaitent améliorer les méthodes pédagogiques appliquées dans les écoles jusqu'à présent, et qui font appel à des ONG pour 
expérimenter, certes, mais en vue de généraliser également sur de plus grandes superficies des méthodes plus efficaces que celles pratiquées dans la plupart des écoles. C'est en collaboration avec les gouvernements que, sur des échelles de dimension grandissante, on peut intervenir. Il existe effectivement des cas de dérive. Comme cela a été dit en préalable, les ONG sont nombreuses et de diverse nature, mais celles que j'évoque interviennent en relation avec les autorités nationales et académiques et pour réaliser les objectifs de ces autorités.

\section{COMMENT GÉNÉRALISER LES INNOVATIONS EXPÉRIMENTÉES?}

SANDRA BARLeT : Parmi les nombreux points importants que nous abordons, il y a en effet celui de la limite des actions des ONG, notamment au regard de la massification et de la durabilité. Les ONG fonctionnent souvent, y compris en matière d'éducation, dans le cadre de financements sous forme de projets. Même si certaines coopérations se distinguent, comme la Suisse, par la durabilité des actions qu'elles mènent, il existe une contrainte de financement à court terme des actions/projets qui est très difficile à maintenir dans le long terme. Comment envisagez-vous ces deux questions centrales, d'une part la capacité de changement d'échelle, d'autre part la durabilité des interventions?

Roland Biache: Aucune ONG, sauf peut-être une ou deux ONG anglo-saxonnes très importantes, n'a prétention à couvrir des territoires nationaux. Les ONG françaises ou latines ne cherchent qu'à bien accompagner des mouvements, des initiatives des pouvoirs publics ou des organisations de base ou de parents à tous les niveaux, du national au local. Je voudrais prendre l'exemple des inspecteurs départementaux de l'éducation nationale au Sénégal. Nous, ONG, avons parfois fait l'erreur d'équiper, voire de suréquiper en ordinateurs des réseaux d'école, alors que l'inspecteur disposait seulement d'une mobylette, de quarante litres d'essence et d'une vieille machine à écrire. Désormais, on pense systématiquement à ces vecteurs essentiels à la bonne marche du système. On a maintenant une vision plus globale. Bernard Dumont a raison de rappeler que la démarche de massification relève du rôle de l'État (et il peut y avoir une forme de pesanteur), et qu'en même temps, il y a des possibilités autres. C'est là que les ONG interviennent, pour mettre en place des actions autour des pédagogies actives auxquelles de nombreux cadres du Sud sont sensibles, pour y avoir été formés avec ou dans nos organisations ou au CIEP. C'est aussi un moyen pour eux de redynamiser les enseignants, qui subissent les pesanteurs administratives inhérentes au système (et je ne parle pas des contraintes 
sociopolitiques des mutations régulières), mais aussi des conditions de travail difficiles, des classes encore surchargées et peu équipées, etc. C'est toute la complexité. Cependant, dans de nombreux pays, je crois que la période forte de tension est passée. Pour favoriser le dialogue, il existe depuis seulement à peine plus de dix ans des "espaces de concertation ", qui réunissent les pouvoirs publics et les ONG. Nous sommes à un moment où aucune ONG ne prétend avoir la solution ni se substituer à l'État dans les pays. Ce n'est ni notre fonction ni notre rôle et je ne vois pas de quel droit nous le ferions. Cela ne veut pas dire que, dans des États dirigés de façon coercitive, nous n'ayons pas à travailler avec ces gouvernements, même si cela ne nous correspond pas, parce qu'il s'agit d'aider et d'accompagner les corps d'inspection, les groupements de femmes, d'enseignants, de parents d'élèves, qui essaient de s'en sortir localement.

Gilbert Migan : Le gouvernement béninois a élaboré des politiques de développement du système éducatif dans lesquelles s'insèrent les activités des ONG intervenant dans l'éducation. Malheureusement, il n'existe pas de concertation entre ces ONG et la structure centrale, le ministère, en vue de mener en synergie le développement du secteur. Cette concertation permettra au niveau central de capitaliser les actions innovantes des ONG dans le système formel de formation. Par exemple, l'ONG Songhaï développe des stratégies de formation et de production qui peuvent être reproduites dans nos écoles de formation formelle, en vue de réaliser une croissance agricole notable. De même, les ONG se plaignent que les formations qu'elles offrent ne bénéficient pas d'une reconnaissance par l'État. Enfin, la concertation peut également permettre de rendre efficace l'intervention des $\mathrm{ONG}$, en les orientant vers les domaines dans lesquels le ministère manque de dynamisme. C'est face à ce vide que le ministère a prévu, dans les jours à venir, la tenue d'un symposium des ONG intervenant dans le système éducatif, en vue de réfléchir à la mise en place d'un cadre de concertation. Il convient de souligner qu'il sera créé incessamment au Bénin un Cadre national de certification $(\mathrm{CNC})$, suite à une rencontre tenue à Abidjan sur le développement des compétences techniques et professionnelles, dans le cadre du Pôle de qualité inter-pays (PQIP). Ce cadre favorisera la reconnaissance des acquis des expériences pour résoudre les problèmes rencontrés par les ONG.

Mahfou Diouf : Je pense qu'il y a deux enjeux. Celui tout d'abord de la capitalisation des expériences. Beaucoup d'initiatives intéressantes ont contribué à mettre en place des offres éducatives. Je prends l'exemple des populations nomades : pendant très longtemps, le système formel ne trouvait pas de modalités pour la scolarisation de leurs enfants. Les ONG ont mis en place des écoles mobiles, ce qui est une innovation très intéressante dans des pays comme le Niger ou le Mali : les écoles se déplacent au rythme des populations, l'enseignant recruté suit les populations, dont les enfants sont ainsi scolarisés. 
La scolarisation universelle ne pourra pas être réalisée seulement au travers des schémas classiques des systèmes d'éducation. Le travail des ONG est d'accompagner ces initiatives locales qui sont agréées par les populations, parce qu'on ne peut pas obliger un individu à choisir un modèle d'éducation qui ne lui convient pas. Dans la formulation des politiques, on ne tient pas toujours compte de l'avis de ces populations et du coup, certaines sont tout à fait réfractaires à ces modèles éducatifs. Par leur souplesse, les ONG peuvent accompagner des dynamiques éducatives en essayant d'aider les populations à les structurer. Je peux citer aussi l'exemple des écoles communautaires de base dans certains pays comme le Sénégal, le Mali, etc., qui ont fait leurs preuves, et qui utilisent la langue nationale et le français. La scolarité dure quatre à cinq années et les enfants peuvent ensuite rejoindre l'éducation formelle, passer des examens, et certains sont à l'université aujourd'hui. Au Sénégal, les premières classes ont été ouvertes entre 1990 et 1996. À la suite de ces initiatives, l'État a été obligé, à travers des financements institutionnels, de mettre en place des dispositifs d'accompagnement. Au Sénégal, c'est le Canada qui a financé les écoles communautaires de base. Les enseignants étaient recrutés par les ONG mais une ONG ne peut payer des enseignants que pour cinq à dix classes, le temps de l'expérimentation. Ensuite, l'État doit prendre le relais. La question qui se pose est aussi celle de la validité scientifique des expériences menées, parce qu'il faut pouvoir prouver leur pertinence et leur capacité à être généralisées. Si l'on met l'accent sur la capitalisation, en associant les universités et les laboratoires de recherche, l'État peut améliorer ses politiques éducatives en partant des expériences menées sur le terrain.

Valérie Huguenin : Le changement d'échelle est une question centrale car les ONG mettent beaucoup d'énergie à développer des innovations, à toucher des publics délaissés par le système classique. Nous, bailleurs, leur demandons à quel moment elles passent le relais, à quel moment elles vont pérenniser. C'est une question difficile aussi bien pour les ONG que pour ceux qui les soutiennent, au-delà du secteur éducatif. Il me semble aussi que la faiblesse des ONG au Sud vient peut-être de la faiblesse de leur structuration collective. Une ONG qui essaie seule de démontrer la plus-value de son expérimentation, quand bien même elle est très compétente, n'a peut-être pas un poids suffisant. En revanche, si les ONG se structurent collectivement, elles seront davantage entendues par leurs interlocuteurs institutionnels, à l'étape de la concertation, et elles pourront peut-être plus facilement proposer ce changement d'échelle, qui est complexe.

Mahfou Diouf : Les partenaires aussi ont un rôle à jouer. Le Projet d'amélioration de l'offre éducative au Sénégal, mis en œuvre en 2000, qui a permis de généraliser les comités de gestion du niveau national au niveau local, et était financé par l'AFD. Nous avions un certain poids par rapport à l'État. 


\section{LE NÉCESSAIRE DIALOGUE ENTRE SOCIÉTÉ CIVILE ET POUVOIRS PUBLICS}

Jacques Marchand : Nous avons évoqué le dialogue de la société civile avec l'État sur les enjeux éducatifs. On peut regretter qu'au Bénin, comme dans d'autres pays, il n'existe pas encore de vraie plateforme de dialogue entre la société civile et l'État, et c'est à renforcer. Dans les pays en développement, les ONG et les associations de solidarité ont un impact très bénéfique sur l'accès à l'école, la qualité de l'éducation, la gestion du système, au niveau local ou national. Il faut évoquer aussi le rôle majeur des ONG dans le plaidoyer en faveur de l'Éducation pour tous, tant au niveau national qu'international, qui est un phénomène marquant de la décennie 2000-2010. On n'aurait pas progressé, depuis dix ans, vers un accroissement significatif de la scolarisation, sans une très forte mobilisation au niveau international, à travers de grandes coalitions d'OSC africaines, européennes, asiatiques, etc. Cela a contribué à une prise de conscience chez les décideurs, qui allouent plus de ressources qu'auparavant, bien que ce soit encore très insuffisant. Cette évolution ne se serait pas produite sans l'aiguillon, la créativité, l'innovation et l'assistance de la société civile au sens large. Au Forum mondial de l'éducation de Dakar en 2000, son rôle a été essentiel. Mais s'il existe, ce dialogue avec les États reste encore à structurer, renforcer, développer. C'est pour cela que, par exemple, l'AFD a financé une Fisong ${ }^{3}$ sur le thème de la gestion locale de l'éducation. Il s'agit de structurer le dialogue entre les sociétés civiles et les ministères de l'Éducation ou les États au sens large, pour améliorer l'accès à l'éducation et la gestion du système. Des progrès ont été réalisés mais il reste beaucoup à faire pour professionnaliser les sociétés civiles au Sud, pour renforcer leur capacité à se structurer, à organiser les informations afin d'être plus percutantes dans le dialogue national sur les politiques éducatives et leur mise en ouvre, à mener dans la durée un dialogue construit sur des objectifs et à procéder par avancées dans le cadre d'une négociation, comme les organisations du mouvement social le font en France. Cela s'apprend, il faut parfois des décennies pour que l'école devienne l'affaire de la société et pas seulement de l'administration. Si l'école française est le produit de très nombreuses interactions liées à l'histoire sociale française, en Afrique, l'école était au départ plutôt issue d'une école coloniale voulue par l'administration, sans implication de la société civile. Ce n'est que tardivement que l'école africaine s'est ensuite progressivement ouverte et imprégnée d'autres influences et des contributions de la société civile. On assiste à un processus d'enrichissement du champ éducatif, avec le temps. Les ONG contribuent à cette évolution, qui

3. Ligne de financement « Facilité d'innovation sectorielle pour les ONG » de l'AFD, qui soutient l'innovation des ONG. 
les a en retour transformées. Souvent, le constat de départ d'une ONG était de dire qu'il n'y avait pas d'école dans tel quartier ou tel village, et qu'il fallait la construire. Maintenant, le constat est infiniment plus complexe, plus intéressant, et porte sur de vrais enjeux. Des questions de structuration sociale, de lutte contre les inégalités, qui existent dans n’importe quelle société, traversent l'école. Faut-il une école pour les riches, ou une école pour tous ? Une école religieuse ou une école laïque? Faut-il une école où les filles pourront pleinement être éduquées, là où, aujourd'hui encore, elles sont souvent marginalisées? Se satisfait-on d'une école médiocre qui abandonne la moitié des enfants en cours de scolarité ou veut-on réellement une école qui accueille, intègre tous les enfants pour une scolarité complète et de qualité ? On est au milieu du gué. Les États peuvent améliorer l'efficacité de leur gestion du système éducatif, ils y sont poussés par la société civile, mais il est clair qu'il faudra mobiliser d'autres acteurs, d'autres ressources, si l'on veut réaliser la scolarisation universelle.

André Gauron : Je partage ce qui vient d'être dit. Si l'on veut progresser aujourd'hui, le dialogue au niveau local avec les parents d'élèves est une mission essentielle. On voit bien, quand on regarde les résultats de la scolarisation au cours des dix dernières années, que ce sont dans les régions les plus rurales, les plus pauvres, où la densité de population est la plus faible, que les retards des scolarisation sont les plus grands, parce que le système est conçu d'en haut, à partir d'une vision d'un monde urbain dans lequel les problèmes des populations rurales, notamment les jeunes filles et le rôle qu'elles jouent au niveau de la cellule familiale, ne sont pas pris en compte. Le système ne pourra pas fonctionner correctement tant qu'il n'y aura pas ce dialogue. Il faut que, y compris au niveau des projets de coopération institutionnelle, cette dimension soit de plus en plus prise en compte.

SANDRA BARLET : Je vais tenter une rapide synthèse des premiers grands axes qui se dégagent. Il y a d'une part un rôle essentiel des ONG pour renforcer le dialogue de la société civile avec l'État et pour faire en sorte que cette société civile participe à la prise de décision. Les ONG jouent également un rôle auprès des institutions dans la lutte contre les inégalités et pour promouvoir l'équité. Les ONG, par leur souplesse, leur capacité à innover, à tester, sont aussi des "têtes chercheuses" de solutions, d'actions, pour lesquelles il faut réfléchir à des modalités d'élargissement. Il faut apprendre de ce qu'ont fait les autres, capitaliser, en associant également des chercheurs, la communauté scientifique.

JACQues Marchand : Je crois qu'il y a un nouveau métier sur lequel les ONG peuvent se positionner, c'est ce dialogue sur les nouveaux dispositifs de formation et d'insertion, ou d'éducation, dans lesquels on retrouve l'État, les organisations professionnelles (du monde rural, ou des zones urbaines), la société 
civile, chacun étant dans sa logique. Il n'existe pas de vraie instance de dialogue, et c'est à l'État de l'organiser, afin que les acteurs arrivent avec des propositions et négocient de telle sorte que le résultat soit un dispositif national. Il y a une technicité de ce type de dialogue. Peu d'ONG disposent de ce savoir-faire.

André Gauron : Il y a des marges de progression énormes dans ce domaine. L'enjeu, ce sont les millions de jeunes qui sont hors du système scolaire, qui ont un avenir professionnel très limité et dont les frustrations peuvent devenir une menace pour la paix et la démocratie. Si l'on ne trouve pas de solutions, on n'ira nulle part. Nous sommes condamnés, ONG, bailleurs, États, etc., à travailler ensemble sur ces questions, car il y a urgence.

Roland Biache : Cela suppose que les bailleurs se coordonnent, ce qui n'est pas le cas partout, et fassent preuve d'un peu d'humilité et d'une capacité à écouter et à respecter les partenaires locaux.

\section{Le PASSAge D'UNE INNOVATION À UNE POLITIQUE PUBLIQUE}

Jacques Marchand: Le bailleur public joue parfois un rôle de " passeur » de l'apport innovant d'une ONG, car les ONG ne sont pas forcément bien considérées par les ministères en Afrique. Il n'y a pas de vraie culture, au sein des appareils d'État, de dialogue et d'écoute des ONG, et il suffit parfois de la présence d'un bailleur pour faire accepter plus facilement une innovation, pour qu'elle s'intègre à la politique éducative. En ce sens, la complémentarité est intéressante.

André Gauron : Si les États considèrent peu les ONG, c'est qu'on s'intéresse rarement à l'organisation du monde éducatif, c'est-à-dire qu'on ne cherche pas à comprendre quels sont les blocages du système éducatif et à quel niveau il faut travailler pour pouvoir le faire évoluer. Les corps d'inspection jouent un rôle très important à cet égard, et quand on se trouve face à des blocages de leur part, on a eu tendance à passer au dessus d'eux, alors que leur rôle est essentiel pour la réussite ou l'échec d'un projet. Très rares sont les actions visant à renforcer leurs capacités et à faire évoluer leurs pratiques. Ce sont des questions essentielles lorsque se pose la question du relais, de la généralisation d'un processus. Je ne parle pas ici des organisations syndicales, le corps enseignant étant le corps le mieux syndiqué dans les pays d'Afrique francophone. La coopération française a tendance à délaisser la gestion et l'évolution du système, alors que c'est une question tout à fait essentielle. On ne peut pas la laisser seulement aux ONG. 
Mahfou Diouf : Il y a aussi la responsabilité des bailleurs de fonds. Je reviens sur le projet de l'amélioration de l'offre éducative. Il existait une convention cadre signée avec le ministère de l'Éducation, des conventions d'objectifs signées avec les inspections et nous avons travaillé avec trois inspections sur tous les aspects. C'est ce qui a permis ensuite au ministère de capitaliser et de généraliser. Je pense que les ONG ont aussi besoin d'être accompagnées. Nous accompagnons d'autres organisations qui ont plus de moyens financiers et qui sont, au-delà de ce financement, des partenaires.

Jacques Marchand : C'est toute la problématique de ce qui a été réalisé avec un projet porté par l'ONG Swisscontact, une association pour la formation et l'insertion des jeunes. Comment faire en sorte que des projets de cet ordre puissent être exploités et repris dans une politique nationale ? Toute la difficulté réside dans le dialogue à mener entre partenaires techniques et financiers du secteur (dont l'AFD au Bénin) et le ministère.

André Gauron : Ce n'était pas seulement un projet Swisscontact, les coopérations française, danoise et allemande intervenaient également. Ce projet avait tous les atouts, au niveau expérimental, pour se transformer et se généraliser. Mais l'inspection de l'enseignement technique n'en voulait pas et effectivement, les responsables du projet n'avaient pas noué les contacts nécessaires avec elle. Lorsque le directeur qui s'en occupait est parti, il a fallu repartir de zéro. C'est un cas très illustratif de ce type de blocages.

Jacques Marchand : J'ai souvent constaté que les innovations proposées par les ONG, même lorsqu'elles suscitent l'intérêt de la direction de l'enseignement, parfois même celui des inspecteurs et des enseignants, doivent, pour être reprises, être intégrées au système d'évaluation dirigé par la direction des examens et des concours, qui est la clé de voûte des systèmes éducatifs. Par exemple, si l'on veut faire une alphabétisation en langue nationale, si l'on veut que soient prises en compte les activités de pratique pédagogique, il faut que cela soit valorisé dans l'examen de fin de cycle primaire. Les enseignants s'impliquent et s'engagent dans les innovations si cela est nécessaire pour l'examen de fin de cycle, puisque c'est lui qui détermine toute l'activité pédagogique du cycle. Donc il faut faire évoluer les examens, si l'on veut que les innovations soient retenues dans un système. Les obstacles sont nombreux, notamment pour les ONG à Cotonou.

André Gauron : Dans le domaine de la formation professionnelle, ce problème ne s'est pas rencontré qu'au Bénin. Une partie des acteurs a cherché à contourner le problème des certifications en développant un examen spécifique, le CQP, certificat de qualification professionnelle. Mais en même temps, on a gardé le CAP, hérité de la tradition française d'enseignement technique, et on se 
retrouve ainsi avec deux examens pour qualifier les mêmes compétences, alors que sur le marché du travail, les artisans ne leur reconnaissent pas la même valeur, le titulaire d'un CQP ayant acquis une meilleure pratique. La question de savoir quel examen doit disparaître n'est pas tranchée, et il est clair que, compte tenu du rôle que joue le CAP pour prolonger éventuellement une scolarité, le mauvais diplôme chasse le bon.

Gilbert Migan : C'est vrai, les CQP et CAP visent tous deux à produire des ouvriers qualifiés pour le marché du travail. Cette situation sera envisagée lors du séminaire de réflexion sur l'élargissement des offres de formation technique et professionnelle qui aura lieu les 13 et 14 octobre 2011 à Cotonou. Il s'agira principalement de revoir les profils d'entrée et de sortie, de même que les certifications. Mais il convient de souligner que les profils d'entrée des dispositifs de formation au CAP et au CQP ne sont pas identiques. Les formés au CAP sont les élèves ayant achevé la classe de $5^{\mathrm{e}}$, tandis que ceux qui entrent en formation au CQP sont des apprentis qui sont en contrat auprès des maîtres-artisans depuis au moins six mois.

\section{ARTICULER \\ LES SYSTÈMES ÉDUCATIFS AVEC LES RÉALITÉS ÉCONOMIQUES}

Bernard Dumont : Notre discussion s'oriente de plus en plus vers le perfectionnement de systèmes éducatifs d'inspiration européenne. On vient d'évoquer, pour la formation professionnelle, ces problèmes de certification. Si l'on raisonne quantitativement dans l'Afrique dite francophone d'aujourd'hui, la plus grande partie de l'économie est encore informelle, et l'important est d'apporter aux agents de l'économie, c'est-à-dire à toute la population, hommes, femmes, jeunes, des compléments de formation leur permettant d'améliorer leur production sans nécessairement passer par le système des examens. Vouloir immédiatement mettre tout le monde dans le système formel est hors de la portée des États, de leur organisation et surtout de leur financement. Il y a une part importante à consacrer au non formel, qui ne doit pas être soumis à ce genre d'impératif. Un certain nombre d'États ont essayé des politiques du non formel avec plus ou moins de bonheur, mais il ne faut pas oublier que les ONG ont encore un champ d'intervention immense, en particulier dans le domaine de la formation professionnelle, surtout agricole. C'est une catastrophe que l'Afrique, centrale et occidentale, en soit réduite aujourd'hui à importer l'essentiel de son alimentation, que le Sénégal importe du riz, le Mali de la viande, et tout cela résulte d'une attention insuffisante portée au perfectionnement des agriculteurs adultes et jeunes dans leur exploitation. Cela ne peut pas passer, dans un délai bref, par le système des examens. Cela relève du non formel. 
André GAUron : Je ne suis pas tout à fait d'accord avec cette définition $\mathrm{du}$ formel et du non formel, qui trouble la compréhension des enjeux. Que ce soit dans le domaine de la formation pour l'artisanat ou l'agriculture, ce qui se développe ne consiste pas à mettre les enfants ou les adultes dans des structures éducatives scolaires. Il y a longtemps qu'on ne le fait plus. Les différentes coopérations ont développé des modes d'apprentissage qui se font souvent en partie sur le terrain, en partie avec des apports théoriques. L'apprentissage dual développé par la coopération allemande et Swisscontact dans le domaine de la formation professionnelle est devenu la norme. Cela montre assez bien qu'on peut développer des structures très légères sans passer par des structures scolaires classiques. Il n'en reste pas moins que, lorsque qu'on est sur le terrain, on voit bien qu'il y a une demande de certification, parce que les gens veulent une reconnaissance sociale, veulent pouvoir montrer le diplôme qui atteste de leur qualification. Cette reconnaissance porte aussi sur la qualité : c'est un moyen de faire reconnaître ses compétences et il importe que ce ne soit pas un diplôme local, comme il l'a été pendant longtemps, un diplôme qui relève de tel groupe d'agriculteurs ou d'artisans, mais qu'il ait une valeur nationale qui puisse permettre de comparer ce que font les uns et les autres. Pour faire cela, il n'est pas nécessaire de mettre les gens dans des systèmes scolaires. Le problème est de savoir si l'on veut un résultat, et de quelle nature. C'est plus compliqué dans le domaine agricole mais avec les organisations professionnelles d'artisans, on a défini des référentiels, le contenu de la certification et les résultats qui doivent être acquis pour obtenir tel type de certification. Toute la difficulté est d'articuler cette logique d'organisation professionnelle avec la structuration académique. Les ONG peuvent jouer un rôle réel notamment en aidant les organisations professionnelles à se structurer localement et nationalement, et en renforçant leurs capacités à prendre en charge les questions de formation. C'est cela le véritable enjeu. Tant qu'on ne réussira pas à articuler cela, on n'obtiendra pas la massification des formes d'apprentissage. Le point de blocage est là.

Alain Dhersigny ${ }^{4}$ : Une catégorie d'acteurs n'a pas été mentionnée : les représentants de la société civile que sont les acteurs du milieu économique, dans le secteur formel mais aussi informel. Les associations de solidarité internationales peuvent aussi contribuer dans ce domaine, parce qu'il s'agit bien des besoins de la société. Il est clair, dans des États africains où la moitié d'une génération doit avoir une formation professionnelle, que les systèmes éducatifs ne sont pas calibrés pour cela et que, pour qu'ils le soient, il faut introduire les acteurs économiques dans la réflexion sur le développement de l'éducation.

4. Alain Dhersigny, qui travaille à la Division éducation et formation professionnelle de l'AFD, a assisté à cette table ronde. 